\title{
Ansiedad y consumo de tabaco en niños y adolescentes
}

\author{
Elisardo Becoña y Ma Carmen Míguez \\ Universidad de Santiago de Compostela \\ Enviar correspondencia a: \\ Elisardo Becoña. Unidad de Tabaquismo. Departamento de Psicología Clínica y Psicobiología. Facultad de Psicología. Campus Universitario Sur. \\ 15782 Santiago de Compostela. E-mail: pcelisar@usc.es
}

\section{RESUMEN}

Cada vez hay mayor evidencia de que existe relación entre fumar cigarrillos y distintos trastornos psicopatológicos, especialmente con depresión y distintos trastornos de ansiedad. Esta evidencia existe en adultos pero se ha estudiado menos en niños y adolescentes. En el presente estudio se analiza en dos muestras representativas de estudiantes de enseñanza primaria ( $N=1.447,11-16$ años) y secundaria ( $N=2.790,14-21$ años) de Galicia la relación existente entre consumo de cigarrillos y ansiedad rasgo. Los resultados indican resultados semejantes en ambas muestras de niños y adolescentes: los que nunca fumaron tienen una puntuación significativamente más baja en ansiedad rasgo que los que fuman. Por sexo, los hombres tienen una menor puntuación en ansiedad rasgo que las mujeres en todas las comparaciones realizadas. Estos resultados indican la consistente relación que hay entre ansiedad y fumar desde edades tempranas hasta la adultez. Se discuten las implicaciones de estos resultados para el tratamiento y la prevención del tabaquismo y de los problemas de ansiedad, como la de analizar si las personas fuman cuando acuden a tratamiento por problemas de ansiedad, y la necesidad de prevenir el consumo de tabaco para con ello prevenir tanto el consumo de tabaco como los posteriores problemas de ansiedad.

\section{ABSTRACT}

There is increasing evidence that a relationship exists between cigarette smoking and several psychopathological disorders, particularly depression and anxiety disorders. This evidence exists in adults but has been little studied in children and adolescents. In this study, we analyse the relationship between cigarette smoking and trait-anxiety in two representative samples of adolescents, in primary education ( $N=1,447,11-16$ years), and in secondary education ( $N=2,790,14-21$ years) in Galicia (Spain). Similar results are found in both samples: those who had never smoked present significantly lower scores in traitanxiety than those who do. Girls scored higher than boys in trait-anxiety, in all the comparisons made. These results indicate the existence of a consistent relationship between anxiety and smoking, which can be observed from an early age to adulthood. The implications of these results for the prevention and treatment of both smoking and anxiety disorders are discussed, as is the implication of taking into smoking antecedents into consideration when a patient seeks anxiety disorder treatment, and the need to prevent smoking consumption and in doing so prevent both smoking and subsequent anxiety disorders.

Key words: smoking, tobacco, anxiety, children, adolescents.

\section{INTRODUCCIÓN}

E incremento en el inicio del consumo de tabaco en jóvenes y adolescentes está llevando a que la prevalencia del consumo de tabaco en la población general se mantenga estable o descienda lentamente, como ocurre en España (Plan Nacional sobre Drogas, 2001). Quienes van dejando de fumar, especialmente a partir de los 40 años, son reemplazados por estos nuevos consumidores.
En los últimos años se ha establecido consistentemente, tanto en estudios de la población general (Farrell et al., 1988; Leonard et al., 2001) como en población psiquiátrica (de León et al., 2002; Hughes et al., 1986), que existe una clara relación entre fumar y padecer en mayor medida distintos trastornos mentales, como depresión, trastornos de ansiedad, trastorno bipolar, etc. A su vez, las personas que padecen trastornos mentales llegan a fumar hasta el doble o el triple (alcohólicos, jugadores patológicos o esquizofré- 
nicos, por ejemplo) que lo que fuman las personas de la población general.

En lo que atañe a los trastornos de ansiedad, distintos estudios muestran consistentemente una clara relación entre su prevalencia y el consumo de tabaco. Una nueva línea de investigación está surgiendo para conocer si el consumo de tabaco previo puede incrementar los trastornos de ansiedad o si, por el contrario, padecer un trastorno de ansiedad lleva a incrementar el consumo de tabaco o a comenzar a fumar.

Estudios como el de Johnson et al. (2000) han encontrado que, quienes a los 16 años fumaban un paquete o más de cigarrillos diariamente, seis años más tarde presentaban un mayor porcentaje de trastornos de ansiedad, respecto a los que a los 16 años sólo fumaban de 1 a 19 cigarrillos diarios. Aunque otros estudios no han replicado este tipo de relación (Patton et al., 1998), los ataques de pánico aparecen cada vez más consistentemente relacionados con fumar, facilitando la conducta de fumar la posterior aparición de ataques de pánico (Breslau y Klein, 1999).

Otra serie de estudios en adolescentes (Audrain et al., 1998; González et al., 1998) encuentran en los fumadores una ansiedad rasgo y una ansiedad estado más alta que en los que sólo han probado los cigarrillos o los que nunca los han probado. Por el contrario, en adultos, no siempre se encuentra esta relación (Becoña et al., 1999). Probablemente la respuesta a esta discrepancia está en que cuando la persona comienza a fumar, la deprivación de tabaco lleva a incrementar el nivel de estrés, convirtiéndose la nicotina en un modulador del humor (Parrott, 1999). Así, fumando la persona reestablece el nivel que para ella es normal de nicotina, pero al mismo tiempo se va estableciendo el proceso de dependencia de la nicotina y una modulación de su ansiedad, estado de ánimo, etc., dependiendo de la disponibilidad o no de nicotina en su organismo; convirtiéndose de esta manera la nicotina en un potente reforzador para el funcionamiento cotidiano del individuo. Se sabe que los fumadores tienen mayor nivel de estrés que los no fumadores (Parrott y Garnham, 1998). Lo anterior viene avalado por el hecho de que, al dejar de fumar, se incrementa ligeramente el nivel de estrés pero, conforme pasa el tiempo, éste disminuye, pasando a ser menor que el que se tenía cuando se fumaba (Cohen y Lichtenstein, 1990; West y Hajek, 1997).

El objetivo del presente estudio es analizar la relación entre ansiedad y fumar en dos muestras representativas de personas escolarizadas de 11 a 21 años de edad para conocer si a estas edades ya aparece esta relación en nuestro medio.

\section{MÉTODO}

Se han utilizado dos muestras. La primera está compuesta por 1.447 estudiantes de enseñanza primaria de Galicia, con edades comprendidas entre los 11 y los 16 años. El $55.1 \%$ eran varones y el $44.9 \%$ mujeres. La muestra se obtuvo aleatoriamente de los centros educativos de enseñanza primaria de Galicia (públicos y privados), estratificada por provincia y tamaño del municipio (más de 50.001 habitantes, de 20.001 a 50.000 habitantes, de 10.001 a 20.000 habitantes, de 5.001 a 10.000 habitantes, y menos de 5.000 habitantes). La muestra fue obtenida en 17 centros de Galicia. La selección de las clases en cada centro se hizo de modo aleatorio cuando en cada centro había más de un aula por curso.

La segunda muestra está compuesta de 2.790 estudiantes de Enseñanza Secundaria Obligatoria de Galicia que estaban cursando el $2^{\circ}$ ciclo de la ESO, BachiIlerato y Formación Profesional, en Institutos (con o sin formación profesional) y en Colegios privados (con o sin formación profesional). Esta muestra fue seleccionada de los centros educativos de Galicia (públicos y privados), mediante un muestreo al azar, estratificada por provincia y tamaño del municipio (más de 50.001 habitantes, de 20.001 a 50.000 habitantes, de 10.001 a 20.000 habitantes, de 5.001 a 10.000 habitantes, y menos de 5.000 habitantes). La muestra fue obtenida en 26 centros de Galicia. La selección de las clases en cada centro se hizo de modo aleatorio cuando en cada centro había más de un aula por curso. La muestra incluye a las personas con edades comprendidas entre 14 y 21 años. Los que tenían más de 21 años $(n=70)$ fueron excluidos del estudio por la gran variabilidad de edades. El $45.6 \%$ eran varones y el $54.5 \%$ mujeres.

Se ha evaluado la ansiedad rasgo mediante la escala del STAIC (Spielberger et al., 1990). La ansiedad rasgo implica la tendencia general a experimentar síntomas de ansiedad a través de una amplia variedad de situaciones estresantes, siendo una características relativamente estable en el individuo. El consumo de tabaco se ha evaluado con varias cuestiones relacionadas con el consumo de tabaco actual (categórico y numérico) y su historia pasada de consumo.

\section{RESULTADOS}

La comparación de los resultados en las categorías de consumo de cigarrillos indica que, en ambas muestras (de 11 a 16 años y de 14 a 21 años), hay diferencias significativas en ansiedad rasgo entre los que nunca fumaron y los otros dos grupos, que son los 
que fumaron alguna vez o los que fuman actualmente $(F(2,1444)=14.07, p<.001$ y $F(2,2787)=16.33, p<$ .001). Los que tienen la menor puntuación en ansiedad rasgo son los que nunca fumaron (ver tabla 1).

La comparación por sexo indica los mismos resultados en ambas muestras: las mujeres tienen significativamente mayor nivel de ansiedad rasgo que los varones $(F(1,1445)=103.24, p<.001 ; 30.65$ vs. 34.56; y $F(1,2788)=264.43, p<.001 ; 33.56$ vs. 38.07); y también tienen mayor puntuación en ansiedad rasgo en cada categoría considerada para el consumo de cigarrillos (nunca, alguna vez, diariamente) (tabla 2), apareciendo también una interacción significativa de las categorías de consumo de cigarrillos por sexo (tabla 3 y figuras 1 y 2).

Al diferenciar a los fumadores por su consumo (no fuma, lo probó alguna vez y fuma diariamente, en los de 11 a 16 años; y en no fuma, fuma de 1 a 10 cigarrillos/día, y fuma más de 10 cigarrillos/día, en los de 14-21 años), se encuentra que los que no fuman tienen el menor nivel de ansiedad rasgo. En los de 1116 años la diferencia es significativa entre los que no fuman respecto a los otros dos grupos. En los de 1421 años aparece una diferencia significativa entre los que no fuman y los que fuman de 1 a 10 cigarrillos/día. Es apreciable que en ambas muestras se incrementa la edad conforme los adolescentes pasan de no fumar a fumar, como también se les incrementa la ansiedad rasgo con la edad (ver tablas 4 y 5). Un análisis independiente de la ansiedad rasgo en función de la edad muestra como la ansiedad se incrementa con la edad en ambas muestras (en la muestra de 11-16 años las medidas de ansiedad rasgo en estas edades son de $30.1,31.7,33.1,33.5,34.0$ y 35.3; y, en la muestra de 14 a 21 años de 34.6, 34.8, 36.0, 36.9, 37.5, descendiendo ligeramente y estabilizándose de 19 a 21 años: $36.5,36.5$ y 36.8$)$.

Tabla 1. Puntuaciones en ansiedad rasgo en función del tipo de consumo de cigarrillos

\begin{tabular}{lcccccc}
\hline & \multicolumn{3}{c}{ Muestra 11-16 años } & \multicolumn{3}{c}{ Muestra 14-21 años } \\
Tipo de fumador & $\mathrm{n}$ & Media & D.T. & $\mathrm{n}$ & Media & D.T. \\
\hline Nunca fumó & 1050 & 31.78 & 7.17 & 1006 & 34.95 & 7.23 \\
\hline Fumó alguna vez & 354 & 33.91 & 8.28 & 1079 & 36.42 & 7.44 \\
\hline Actualmente fuma & 43 & 35.27 & 7.82 & 705 & 36.90 & 8.29 \\
\hline Total & 1447 & 32.40 & 7.54 & 2790 & 36.01 & 7.63 \\
\hline \multicolumn{3}{c}{$\mathrm{F}(2,1444)=14.07, \mathrm{p}<.001$} & $\mathrm{~F}(2,2787)=16.33, \mathrm{p}<.001$ \\
Scheffé: $1-2(\mathrm{p}<.001) ; 1-3(\mathrm{p}<.05)$ & $1-2(\mathrm{p}<.001), 1-3(\mathrm{p}<.001)$ \\
\hline
\end{tabular}

Tabla 2. Diferencias por sexo en ansiedad rasgo en función del consumo de tabaco

\begin{tabular}{lcccc}
\hline \multicolumn{3}{c}{ Muestra 11-16 años } & \multicolumn{2}{c}{ Muestra 14-21 años } \\
Consumo de tabaco & Media & D.T. & Media & D.T. \\
\hline $\begin{array}{l}\text { Nunca } \\
\quad \text { Hombre }\end{array}$ & 30.35 & 6.61 & 33.24 & 6.88 \\
$\quad$ Mujer & 33.43 & 7.43 & 36.79 & 7.15 \\
\hline $\begin{array}{l}\text { Alguna vez } \\
\quad \text { Hombre }\end{array}$ & 31.26 & 7.38 & 33.70 & 6.59 \\
$\quad$ Mujer & 38.02 & 7.94 & 38.50 & 7.39 \\
\hline Diariamente & & & & \\
$\quad$ Hombre & 32.33 & 6.24 & 33.9 & 18.31 \\
$\quad$ Mujer & 37.40 & 8.26 & 38.93 & 7.64 \\
\hline Hombre & 30.65 & 6.83 & 33.56 & 7.12 \\
Mujer & 34.56 & 7.82 & 38.07 & 7.43 \\
& $F(1,1445)=103.24, p<.001$ & $F(1,2788)=264.43, p<.001$ \\
\hline
\end{tabular}

Tabla 3. Resultados del análisis de varianza $(F)$ de las puntuaciones de ansiedad rasgo en función del consumo de tabaco (nunca, alguna vez, diariamente) y sexo (hombre, mujer)

\begin{tabular}{lccc}
\hline & $\begin{array}{c}\text { Consumo } \\
\text { de tabaco }\end{array}$ & Sexo & $\begin{array}{c}\text { Interacción de consumo } \\
\text { de tabaco } x \text { sexo }\end{array}$ \\
\hline $\begin{array}{l}\text { Muestra } \\
11-16 \text { años }\end{array}$ & 2.43 & $10.15^{*}$ & $8.54^{* * *}$ \\
\hline $\begin{array}{l}\text { Muestra } \\
14-21 \text { años }\end{array}$ & 3.34 & $91.22^{* *}$ & $2.76^{*}$ \\
\hline${ }^{*} p<05^{* *}$ * $<01 * * * 01$ &
\end{tabular}

Tabla 4. Ansiedad rasgo y edad en función del consumo de tabaco, muestra de 11 a 16 años

\begin{tabular}{lcccccc}
\hline & \multicolumn{4}{c}{ Ansiedad-rasgo } & \multicolumn{2}{c}{ Edad } \\
Consumo de tabaco & $\mathrm{n}$ & $\%$ & Media & D.T. & Media & D.T \\
\hline No fuma & 1050 & 72.6 & 31.78 & 7.17 & 12.55 & 1.11 \\
Lo probó alguna vez & 354 & 24.5 & 33.91 & 8.28 & 13.20 & 1.12 \\
Fuma diariamente & 43 & 3.0 & 35.27 & 7.82 & 14.16 & 0.95 \\
\hline $\mathrm{F}(2,1444)=$ & \multicolumn{1}{c}{$14.07^{* * *}$} & \multicolumn{3}{c}{$81.50^{* * *}$} \\
Scheffé: & & $1-2,1-3$ & $1-2,1-3,2-3$ \\
\hline
\end{tabular}

Tabla 5. Ansiedad rasgo y edad en función del consumo de tabaco, muestra de 14 a 21 años

\begin{tabular}{lcccccc}
\hline & \multicolumn{4}{c}{ Ansiedad-rasgo } & \multicolumn{2}{c}{ Edad } \\
Consumo de tabaco & $\mathrm{n}$ & $\%$ & Media & D.T. & Media & D.T \\
\hline No fuma & 1956 & 70.1 & 35.66 & 7.36 & 16.17 & 1.66 \\
Fuma 1-10 cig./día & 664 & 23.8 & 37.02 & 8.14 & 16.94 & 1.76 \\
Fuma > 10 cig./día & 170 & 6.1 & 36.13 & 8.34 & 17.53 & 1.65 \\
\hline $\mathrm{F}(2,1444)=$ & & & $7.81^{* * *}$ & $89.13^{* * *}$ \\
Scheffé: & & & $1-2$ & $1-2,1-3,2-3$ \\
\hline
\end{tabular}




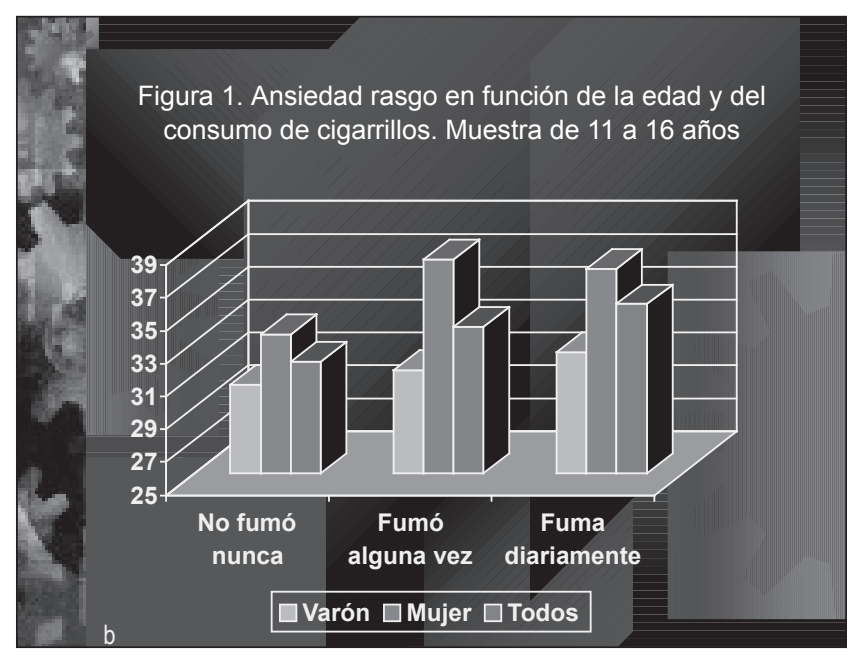

\section{DISCUSIÓN}

Los resultados obtenidos en este estudio, con dos muestras distintas, representativas de los niños y adolescentes escolarizados de 11 a 21 años en Galicia, sugieren que los que no fuman tienen una menor ansiedad rasgo que los que han probado los cigarrillos o los que fuman actualmente. Este hecho va en la línea de otros estudios que han evaluado la ansiedad rasgo en relación con fumar (Audrain et al, 1998; González et al., 1998; Foreyt et al., 1997). En nuestra muestra, aunque no de forma significativa, quienes fuman pocos cigarrillos tienen un mayor nivel de ansiedad que los que tienen un mayor consumo de cigarrillos, en la muestra de 14 a 21 años.

Aunque el presente estudio es descriptivo, sus datos apuntan a lo que se viene afirmando en otros estudios acerca de que comenzar a fumar conduce a distintas alteraciones en el comportamiento (como estrés, ansiedad o cambios en el estado de ánimo), que el individuo nota al estar deprivado de la nicotina, y que solventa fumando. Al incrementársele el nivel de dependencia de la nicotina (y el consumo de cigarrillos) consigue estabilizar estos efectos negativos, dadas las propiedades ansiolíticas de la nicotina (Comeau et al., 2001). También podría haber otra explicación alternativa, consistente en que descubren que con la nicotina pueden incrementar o disminuir su nivel de activación (Grobe y Perkins, 2000), convirtiéndose así fumar en la herramienta más útil que tienen para manejar su ansiedad y con una clara función reforzante. De ser esto cierto, los adolescentes tendrían un problema adicional a largo plazo: al intentar dejar de fumar se les exacerbarían los síntomas, dificultándoles la abstinencia, al menos en los primeros días. Si consiguen mantenerse abstinentes varios meses, entonces su nivel de ansiedad disminuye (Becoña et al., 2002); de ahí que quienes tienen mayores niveles de ansiedad les cuesta más dejar de fumar (Cinciripini et al., 1995). Curiosamente, esta relación no siempre se

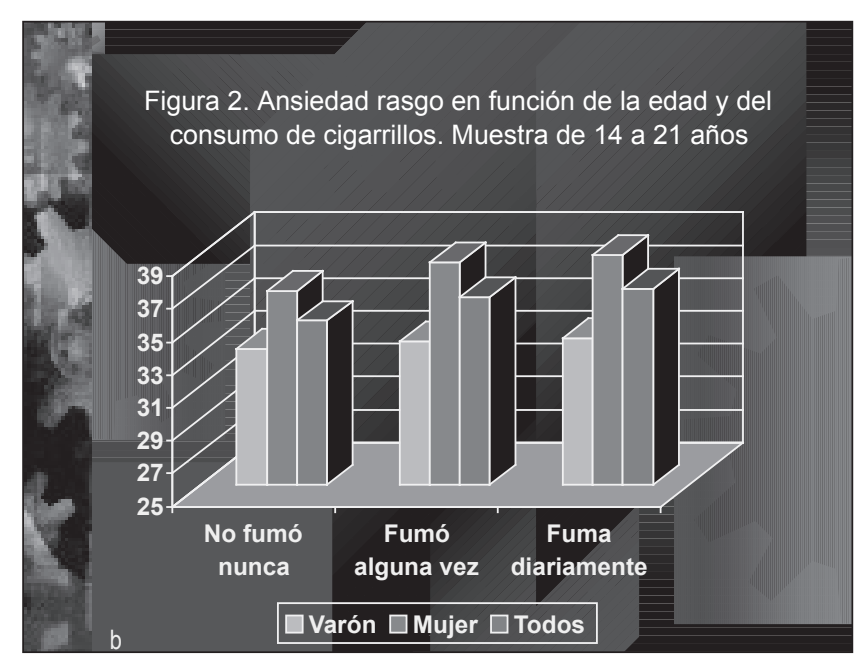

encuentra en la población adulta: en algunos estudios sí (Kandel et al., 2001), pero no en otros (Becoña et al., 1999; Takemura et al., 1999).

El resultado obtenido del mayor nivel de ansiedad en el nivel de consumo de 1 a 10 cigarrillos/día confirma lo que sabemos de que cuando los adolescentes comienzan a fumar se les incrementa el estrés (Parrott, 1999; USDHHS, 1988). Esto tiene claras implicaciones para la prevención del consumo de tabaco. Medidas restrictivas para el consumo en las escuelas e institutos, restricción y control de la publicidad dirigida a ellos, control de la venta de cigarrillos sueltos, detección por parte de los padres de su consumo, junto a adecuados programas preventivos, en la escuela, la familia y los medios de comunicación, evitaría que muchos de ellos no se hiciesen dependientes y que no desarrollasen otros problemas físicos o psicopatológicos relacionados con fumar cigarrillos.

Las mayores puntuaciones que encontramos en ansiedad rasgo en las mujeres respecto a los varones aparecen sistemáticamente en los estudios con esta escala, como también que los fumadores puntúen más que los no fumadores en la misma (Forgays et al., 1993).

La evidencia indica que prevenir el consumo de tabaco en la adolescencia previene también que algunos de estos que serían fumadores no desarrollen trastornos de ansiedad en su vida adulta (Johnson et al., 2000). En el estudio de Patton et al. (1998) encuentran que tanto el fumar de los iguales como la depresión y la ansiedad son predictores de comenzar a fumar, en adolescentes de 14 y 15 años de edad; estos autores llegan a sugerir la existencia de una relación causal entre tener depresión, ansiedad y comenzar a fumar; sin embargo, no encuentran un apoyo consistente de que la ansiedad y la depresión lleven a una progresión en el consumo diario. En nuestro estudio el hecho de que la ansiedad rasgo se incremente con la edad no permite llegar a una conclusión clara y contundente sobre la existencia de una relación de causalidad entre 
fumar y ansiedad rasgo. Sí parece claro que la tolerancia a la nicotina va incrementándose con la edad, como lo indica un mayor consumo de cigarrillos con la edad $y$, al mismo tiempo, un incremento de la ansiedad rasgo con la edad. Futuros estudios, especialmente longitudinales, deben analizar específicamente esta asociación para conocer si es una mera relación, una relación modulada por otra u otras variables, o una relación de causalidad ansiedad-fumar o fumar-ansiedad. Aún así, las implicaciones de estos resultados cara al tratamiento y la prevención, tanto del tabaquismo como de los trastornos de ansiedad, son claros por la interacción que existe entre fumar, ansiedad, estrés y otras características relacionadas con el estado de ánimo de las personas.

\section{AGRADECIMIENTOS}

Esta investigación ha sido financiada mediante los proyectos XUGA2110B96 y XUGA21105B98 de la Secretaría de Investigación y Desarrollo de la Xunta de Galicia.

\section{REFERENCIAS}

Audrain, J., Lerman, C., Gomez-Caminero, A., Boyd, N. R.y Orleans, C. T. (1998). The role of trait anxiety in nicotine dependence. Journal of Applied Biobehavioral Research, 3, 29-42.

Becoña, E., Vázquez, F., Fuentes, M. C. y Lorenzo, M. C. (1999). Anxiety, affect, depression and cigarette consumption. Personality and Individual Differences, 26, 113-119.

Becoña, E., Vázquez, F.L. y Míguez, M.C. (2002). Smoking cessation and anxiety in a clinical sample. Personality and Individual Differences, 32, 489-494.

Breslau, N. y Klein, D. F. (1999). Smoking and panic attacks. An epidemiological investigation. Archives of General Psychiatry, 56, 1141-1147.

Cinciripini, P. M., Lapitzky, L., Seay, S., Wallfisch, A., Meyer, W. J. y van Vunakis, H. (1995). A placebo-controlled evaluation of the effects of buspirone on smoking cessation: Differences between high- and low-anxiety smokers. Journal of Clinical Psychopharmacology, 15, 182-191.

Cohen, S. y Lichtenstein, E. (1990). Perceived stress, quitting smoking, and smoking relapse. Health Psychology, 9, 466-478.

Comeau, N., Stewart, S. H. y Loba, P. (2001). The relations of trait anxiety, anxiety sensitivity, and sensation seeking to adolescents'motivation for alcohol, cigarette, and marijuana use. Addictive Behaviors, 26, 803-825.

de Leon, J., Becoña, E., Gurpegui, M., González-Pinto, A. y Díaz, F. J. (2002). The association between high nicotine dependence and severe mental illness may be consistent across countries. Journal of Clinical Psychiatry, 63, 812-816.

Farrell, M., Howes, S., Taylor, C., Lewis, G., Jenkins, R., Bebbington, P., Jarvis, M., Brugha, T., Gill, B. y Meltzer, H. (1998). Substance misuse and psychiatric comordibidy: An overview of the OPCS National Psychiatric Morbidity Survey. Addictive Behaviors, 23, 909-918.

Foreyt, J. P., Goodrick, G. K., Schaefer, C., Jackson, A. S., Squires, W. G. y Poston, W. S. C. (1997). Personality characteristics of current and former smokeless tobacco users. American Journal of Health Behavior, 21, 299-309.

Forgays, D. G., Bonaiuto, P., Wrzesniewski, K. y Forgays, K. (1993). Personality and cigarette smoking in Italy, Poland, and the United States. International Journal of Addictions, 28, 399-413.

González, C., Navarro, J. I. y Salvador, L. (1998). Factores psicosociales que intervienen en la adquisición del comportamiento tabáquico. Revista Latinoamericana de Psicología, 30, 49-66.

Grobe, J. E. y Perkins, K. A. (2000). Behavioral factors influencing the effects of nicotine. En M. Piasecki y P.A. Newhouse (Eds.). Nicotine in psychiatry (pp. 59-81). Washington, DC: American Psychyiatric Press.

Hughes, J. R., Hatsukami, D. K., Mitchell, J. E. y Dahlgren, L. A. (1986). Prevalence of smoking among psychiatry outpatients. American Journal of Psychiatry, 143, 993997.

Johnson, J. G., Cohen, P., Pine, D. S., Klein, D. F., Kasen, S. y Brook, J. S. (2000). Association between cigarette smoking and anxiety disorders during adolescence and early adulthood. JAMA, 284, 2348-2351.

Kandel, D. B., Huang, F. Y. y Davies, M. (2001). Comorbidity between patterns of substance use dependence and psychiatric syndromes. Drug and Alcohol Dependence, 64, 233-241.

Leonard, S., Adler, L. E., Benhammou, K., Berger, R., Breese, C. R., Drebing, C., Gault, J., Lee, M. J., Logel, J., Olincy, A., Ross, R. G., Stevens, K., Sullivan, B., Vianzon, R., Virnich, D. E., Waldo, M., Walton, K. y Freedman, R. (2001). Smoking and mental illness. Pharmacology, Biochemistry and Behavior, 70, 561-570.

Parrott, A. C. (1999). Does cigarette smoking cause stress? American Psychologist, 54, 817-820.

Parrott, A. C. y Garnham, N. J. (1998). Comparative mood states and cognitive skills of cigarette smokers, deprived smokers and nonsmokers. Human Psychopharmacology, 13, 367-376.

Patton, G. C., Carlin, J. B., Coffey, C., Wolfe, R., Hibbert, M., Bowes, G. (1998). Depression, anxiety, and smoking initiation: a prospective study over 3 years. American Journal of Public Health, 88, 1518-1522.

Plan Nacional sobre Drogas (2001). Observatorio español sobre drogas. Informe $n^{\circ} 4$. Madrid: Delegación del Gobierno para el Plan Nacional sobre Drogas.

Spielberger, C. D., Edwards, C. D., Lushene, R. E., Montuori, J. y Platzek, D. (1990). STAIC. Cuestionario 
de autoevaluación ansiedad estado-rasgo en niños. Madrid: TEA.

Takemura, Y., Akanuma, M., Kikuchi, S. y Inaba, Y. (1999). Cross-sectional study on the relationship between smoking or smoking cessation and trait anxiety. Preventive Medicine, 29, 496-500.
U. S. D. H. H. S. (1988). Nicotine addiction. A report of the Surgeon General. Rockville, MD: U. S. Department of Health and Human Services.

West, R. y Hajek, P. (1997). What happens to anxiety levels on giving up smoking? American Journal of Psychiatry, 154, 1589-1592. 